\title{
Programa de control de calidad
}

\author{
Ricardo Borda M.; Daniel De León; Jorge Michelsen*
}

RESUMEN: El objetivo de este proyecto busca mejorar el nivel de atención médica de nuestros pacientes, optimizando los recursos económicos de ellos. Se trata de un estudio descriptivo prospectivo cuya población son las pacientes que ingresan al Departamento. Se crearon unas bases de datos que permitieron de una manera prospectiva y con un protocolo establecido, evaluar en cualquier momento las actividades clínicas de nuestro Departamento; con este fin se utilizaron los criterios y marcadores de calidad establecidos por el Colegio Americano de Obstetricia y Ginecología y las pautas del Departamento de Obstetricia y Ginecología del Hospital Universitario de San Ignacio. Se revisaron 2349 procedimientos distribuidos en 1820 pertenecientes al servicio de Obstetricia y 529 correspondientes al servicio de Ginecología, encontrando 241 marcadores positivos en Obstetricia y 69 en Ginecología. El marcador de calidad que más se presentó en Ginecología fue diagnóstico prequirúrgico diferente al postquirúrgico que representó el $51 \%$ y en Obstetricia fue admisión de recién nacido a término en la unidad de recién nacidos que representó el $60 \%$. En la evaluación del comité de calidad en el área de ginecología se encontraron 6 conductas inadecuadas y 6 conductas con oportunidad de mejorar la atención, sobre las cuales se tomaron las medidas del caso.

PALABRAS CLA VES: Calidad, marcador de calidad.

SUMMARY: The objetive of this project is improve the medical attention level in our patients. This is a prospective, descriptive study. The population are all the patients that were admitted in the service. We created a date bases that permit on prospective and with an established protocolo, evaluate in any moment the clinical activities of the service.

With this purpose we used the set criterios and the quality indicators established by The American College of Gynecology and Obstetric and the guidelines of the San Ignacio Gynecology and Obstetric Department.

We reviewed 2349 procedures distributed like this: 1820 of the Obstetric service and 529 of the Gynecology service, found 241 indicators positives of the Obstetric service and 69 of Gynecology service.

The mont repested clinical indicator in Gynecology was prequirurgical different diagnostic that the post quirurgical diagnostic represented by the $51 \%$; in Obstetric was newborns admitted in the newborn unit with the $60 \%$. In the evaluation of the quality control comitte in the Gynecology area we found six inadecuate actions and six actions with opportunities for omprove the attention. We made corrections about those actions.

KEY WORDS: Quality, clinical indicators.

\section{Objetivo}

Uno de los objetivos más importantes del Departamento de Ginecología y Obstetricia del Hospital San Ignacio es garantizar una atención médica de calidad óptima. Previamente, se utilizaba como herramienta la revisión de historias clínicas, la revisión de las complicaciones, etc. Hoy en día se recomienda utilizar herramientas más modernas, que miden de una manera más directa la calidad de atención brindada a los pacientes.

El concepto de CALIDAD TOTAL busca garantizarle al usuario calidad en el servicio que se le está prestando. Este nuevo concepto implica una mayor calidad como proceso dinámico y continuo.

\footnotetext{
* Hospital San Ignacio. Departamento de Obstetricia y Ginecología.
} Pontificia Universidad Javeriana. Santa Fe de Bogotá
La idea de CALIDAD TOTAL no es nueva. Después de la Segunda Guerra Mundial un estadista de MIT (Massachusetts Institute of Tecnology) llamado Edward Deming ya hablaba de la necesidad de una calidad total, previniendo los defectos en lugar de corregirlos después de que ya habían ocurrido. Argumentaba que el último enfoque era ineficiente y que a largo plazo era más efectivo prevenir los errores que corregirlos. Por esto, la calidad debe ser vista como una adecuación permanente a las expectativas del usuario. Este proyecto busca mejorar el nivel de atención médica de nuestros pacientes, optimizando los recursos económicos de ellos.

\section{Materiales y métodos}

Se trata de un estudio descriptivo prospectivo cuya población son las pacientes que ingresan al Departamento de Obstetricia y Ginecología del Hospital Universita- 
rio de San Ignacio, cualesquiera que sea su origen incluyendo los Servicios generales, Pensionados y Seguro Social.

Calidad de la atención médica se define como la habilidad para obtener las metas deseables (estados de salud) usando medios legítimos (procedimientos o cursos de acción en atención en salud).

Se diseñó un sistema de información basado en la plataforma Windows utilizando Access de Microsoft como manejador de base de datos. Basados en lo anterior se crearon los programas que mensualmente procesan la información obteniéndose un informe de las actividades de las siguientes áreas:

a. Resumen Final de Obstetricia.

b. Resumen Final de Ginecología.

c. Procedimientos Obstétricos de Sala de Partos.

d. Procedimientos no obstétricos de Sala de Partos.

\section{a. Resumen final de Obstetricia}

En él se incluye la información básica de cada paciente. En caso que haya tenido su parto, se utilizará la base de datos de Procedimientos Obstétricos de Sala de Partos para obtener cualquier información que se requiera. $\mathrm{Al}$ final de este resumen, se encuentran los marcadores de calidad que se utilizan como banderas para evaluar aquellos casos problema. Ver anexo 1 .

\section{b. Resumen final de Ginecología}

En él se incluye la información básica de cada paciente, (Historia clínica, diagnóstico de ingreso, diagnóstico de egreso, diagnóstico de patología, etc.) a partir de la cual, se obtendrán las estadísticas de esta área. $\mathrm{Al}$ final de este resumen, se encuentran los marcadores de calidad correspondientes. Ver anexo 2.

\section{c. Procedimientos Obstétricos de Sala de Partos}

Esta base de datos incluye la información básica clínica de las pacientes, recién nacidos y procedimientos Obstétricos relacionados a Partos. Esta base de datos servirá de apoyo a la de Resumen Final de Obstetricia.

\section{d. Procedimientos no obstétricos de Sala de Partos}

Esta base de datos incluye la información básica clínica de las pacientes a quien se les ha practicado algún procedimiento en sala de Partos diferente de la atención del parto o cesárea.

Las historias clínicas que se revisaron por tener cualquiera de los marcadores positivos se presentaron al comité de calidad del Departamento para que luego con todos los integrantes del mismo evaluaran los casos y concluyan si las conductas y/o complicaciones evaluadas son adecuadas a los estándares de práctica médica aceptada o si requiere algún tipo de correctivo. Si la conducta no fue la adecuada, el comité debe sugerir los pasos a seguir para corregir este problema.

La información fue digitada en la computadora por una misma persona que fue entrenada para esta labor, donde los autores revisando lo comprendido entre los meses de julio a diciembre de 1993. Previamente se tomó un período de prueba de 6 meses para estandarizar y evaluar la confiabilidad de la información alimentada al computador, comparando con los registros médicos del Servicio de Estadística del Hospital Universitario de San Ignacio.

Se creó el comité de control de calidad encargado de la evaluación de los marcadores de calidad positivos, teniendo como parámetros los criterios del Colegio Americano de Obstetricia y Ginecología además de las normas de manejo del Departamento de Ginecología y Obstetricia del Hospital Universitario de San Ignacio. Este análisis se consigna en un formato preestablecido. Ver anexo 3.

\section{Resultados}

Se revisaron los marcadores de calidad de los procedimientos obstétricos y no obstétricos realizados en 2349 pacientes en total (ver cuadro 1). En ginecología se encontraron un total de 69 marcadores de calidad positivos entre 529 casos discriminados en el cuadro 2; de obstetricia 241 marcadores de calidad positivos entre 1820 casos obstétricos discriminados en el cuadro 3.

\section{Cuadro 1}

RELACION ENTRE NUMERO DE CASOS Y MARCADORES DE CALIDAD

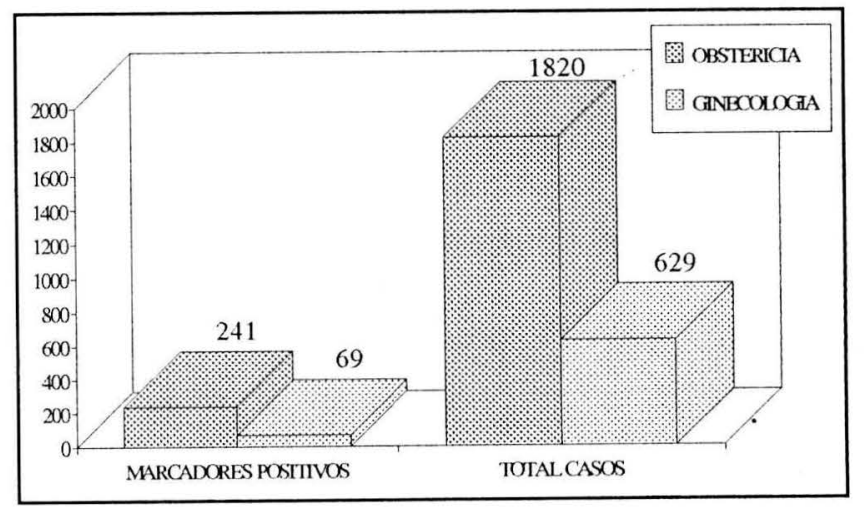

Cuadro 2

DISTRIBUCION PORCENTUAL DE LOS MARCADORES DE CALIDAD POSITIVOS DEL AREA GINECOLOGICA

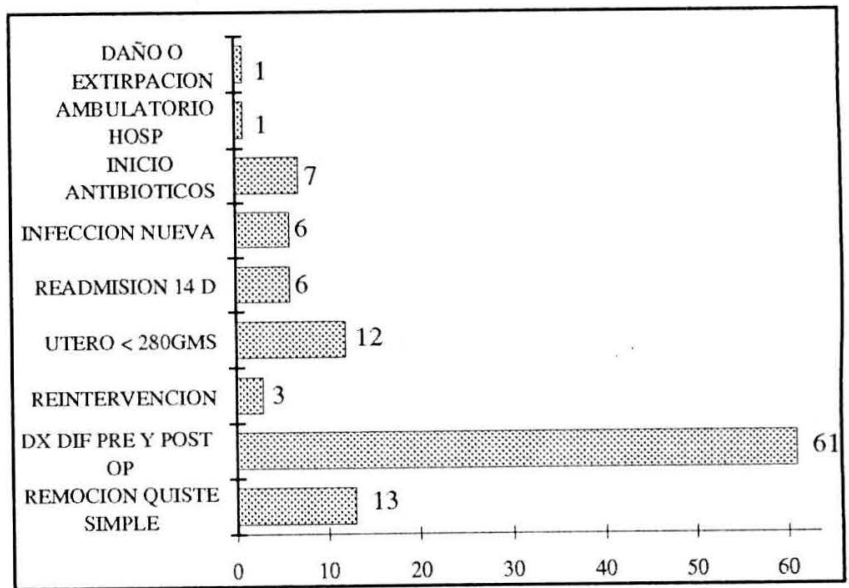


DISTRIBUCION PORCENTUAL DE LOS MARCADORES DE CALIDAD POSITIVOS EN EL AREA DE GINECOLOGIA

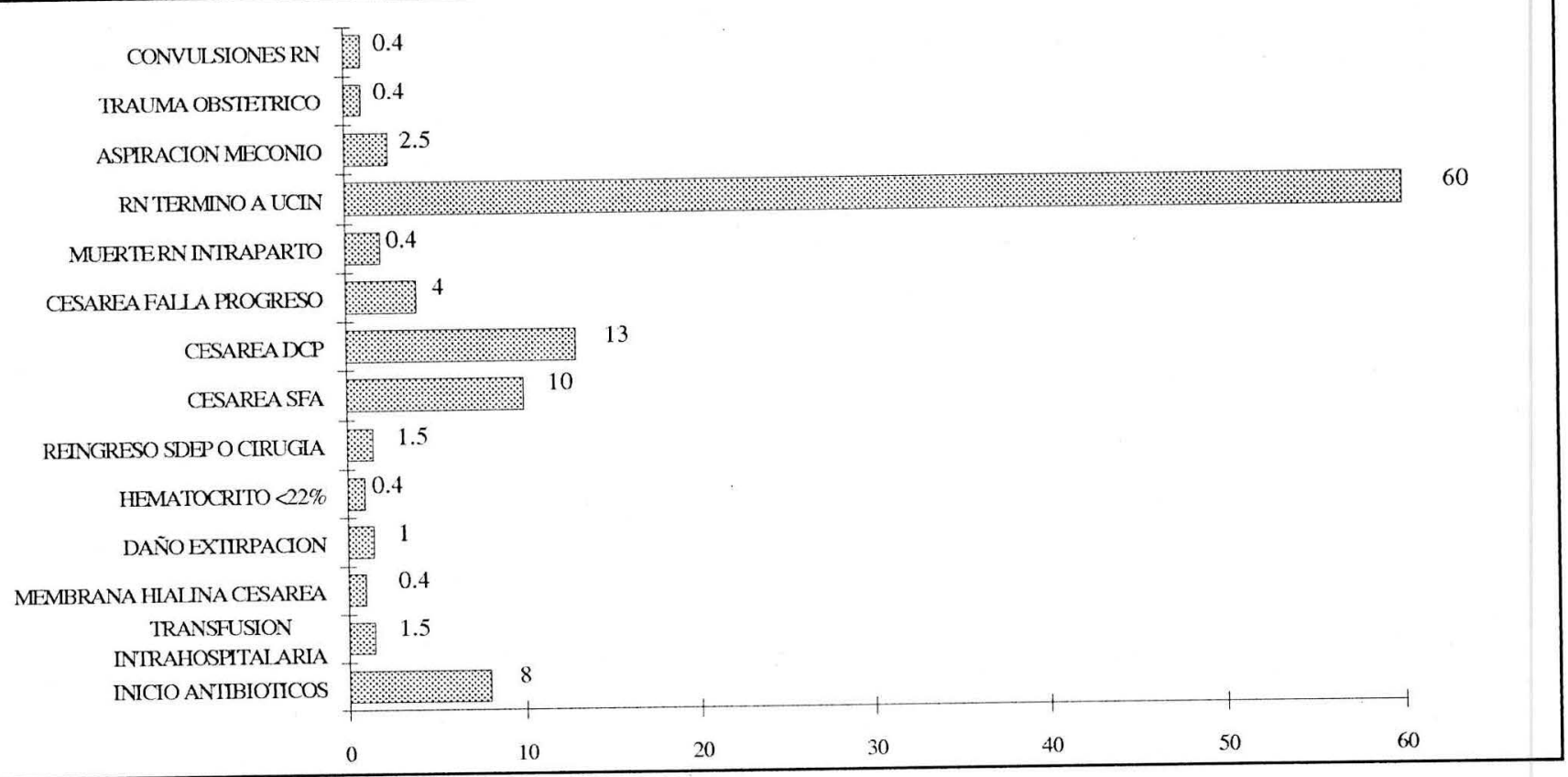

\section{Conclusión}

La profesión médica tradicionalmente ha dedicado parte de sus energías en un control interno, autodeterminado, de la calidad de la atención médica prestada a los pacientes. Esto se ha hecho tradicionalmente por medio de reuniones de morbilidad, mortalidad y requisitos de certificación para procedimientos y especialidades. Este enfoque es característicamente "curativo" sin darle importancia al enfoque preventivo.

La implementación y realización de este programa de control de calidad en el Departamento de Obstetricia y Ginecología del Hospital Universitario de San Ignacio ha permitido conocer, evaluar y corregir en forma dinámica las conductas no adecuadas y las complicaciones más frecuentemente cometidas en la atención de las usuarias del departamento.

Se trata de un programa que se seguirá aplicando y perfeccionando en el departamento y que podría ser aplicado en todos los Departamentos del Hospital Universitario de San Ignacio y en general en todos los servicios de Obstetricia y Ginecología de los Hospitales de Colombia.

El marcador de calidad que más se detectó en ginecología fue diagnóstico de ingreso diferente al diagnóstico de egreso correspondiendo al 51\% de los casos, y en Obstetricia fue traslado de recién nacido a término a la unidad de recién nacidos correspondiendo al $60 \%$ de los casos.

El comité de calidad encargado de la evaluación de los casos con marcadores de calidad positivos siguiendo el protocolo de evaluación encontró en el área de ginecología:

\begin{tabular}{|l|c|}
\hline \multicolumn{1}{|c|}{ Tipo de atención } & Número (porcentaje) \\
\hline Atención adecuada & $57(82.6 \%)$ \\
\hline Atención inadecuada & $6(8.7 \%)$ \\
\hline Oportunidad mejorar atención & $6(8.7 \%)$ \\
\hline
\end{tabular}

En los casos problema, no se presentaron complicaciones médicas para las pacientes.

Las medidas tomadas para evitar que se vuelvan a presentar estos casos incluyeron:

- Mejorar elaboración de las historias clínicas.

- Mejorar la evaluación de las pacientes por parte de la Junta Quirúrgica.

- Incrementar el nivel de supervisión en los casos que ingresan por el servicio de urgencias.

- Evitar las inconsistencias entre la historia clínica y las bases de datos de la computadora, por medio de la evaluación y el entrenamiento permanente de la digitadora.

En cuanto al área de obstetricia, se encontró un número que el $60 \%$ de los marcadores de calidad positivos correspondían a recién nacidos a término admitidos a la Unidad de recién Nacidos. Por lo tanto, fue necesario crear un comité conjunto entre el departamento de pediatría y de obstetricia para analizar estos casos y determinar las medidas a tomar.

Es importante resaltar, que algunos marcadores de calidad no se presentaron durante este período y son los siguientes:

- Paro Cardiorespiratorio

- Eclampsia

- Membrana hialina secundaria a Cesárea o Inducción. 
Como beneficios adicionales de este programa, se alcanzaron los siguientes logros: 1) Automatizar la generación de las estadísticas mensuales.

2) Implementar los Marcadores de Calidad en las áreas de $\mathrm{O}$ y $\mathrm{G}$, con protocolos de manejo estandarizados $y$ evaluados con una periodicidad anual.

3) Crear bases de datos de las patologías más frecuentes para comenzar a adquirir una experiencia "propia" del departamento evaluable estadísticamente.
4) Involucrar a todo el personal del Departamento, tales como aseadoras, auxiliares de enfermería, profesores, etc., en un programa de Mejoramiento continuo a través de conferencias, evaluaciones con los distintos estamentos del Departamento.

5) Crear bases de datos como "archivo docente" de patologías poco frecuentes.

6) Tener bases de datos que permitan evaluar la factibilidad de los proyectos de investigación que se propongan.

\section{BIBLIOGRAFIA}

1. Quality Improvement in Health Care. Curr Prob Obstet. Gynecol. Fertil. Sept-Oct. 1991; 151-174.

2. Donabedian A. The Quality of Care: How can it be assessed? JAMA 1988; 260: 1743-1748.

3. Gambone JC., Reiter RC., Lench JB. Quality Assurance Indicators and short term outcome of hysterectomy. Obstet. Gynecol. 1990; 76: 841-845.
4. Silberman L. Quality Assurance in Obstetrics: A model Obstet. Gynecol. 1990; 76: 466-470.

5. American College of Obstetricians and gynecologists: Quality Assurance in Obstetrics and Gynecology. Washington DC, American College of Obstetricians and gynecologists, 1989. 